\title{
On Construction of a Super Hierarchy of the Wadati-Konno- Ichikawa Equation
}

\author{
Xuemei Li $($ ib) and Lutong $\mathrm{Li}$ \\ School of Mathematics and Statistics, Zhengzhou University, 100 Kexue Road, Zhengzhou 450001, China \\ Correspondence should be addressed to Xuemei Li; lixuemei@zzu.edu.cn
}

Received 8 December 2019; Revised 18 January 2020; Accepted 20 January 2020; Published 7 February 2020

Academic Editor: Marianna Ruggieri

Copyright (c) 2020 Xuemei Li and Lutong Li. This is an open access article distributed under the Creative Commons Attribution License, which permits unrestricted use, distribution, and reproduction in any medium, provided the original work is properly cited.

In this paper, a super Wadati-Konno-Ichikawa (WKI) hierarchy associated with a $3 \times 3$ matrix spectral problem is derived with the help of the zero-curvature equation. We obtain the super bi-Hamiltonian structures by using of the super trace identity. Infinitely, many conserved laws of the super WKI equation are constructed by using spectral parameter expansions.

\section{Introduction}

The super extensions of the standard integrable systems in two-dimensional spacetime have been investigated for the recent several decades. Many classical integrable equations have been extended to be the super completely integrable equations, such as the super Korteweg-de Vries (KdV) equation [1-3], super AKNS [4-7], super Kadomtsev-Petviashvili (KP) [8], super Kaup-Newell (KN) [9], super Camassa-Holm $(\mathrm{CH})$ [10], super vector nonlinear Schrödinger equations [11], super Heisenberg [12], and so on [13-20].

The Wadati-Konno-Ichikawa (WKI) equation, proposed in [21], can be written in the form

$$
\begin{aligned}
& i u_{t}=\left(\frac{u}{\sqrt{1+u v}}\right)_{x x}, \\
& i v_{t}=-\left(\frac{v}{\sqrt{1+u v}}\right)_{x x},
\end{aligned}
$$

which can be used to describe the nonlinear oscillation of elastic beam under tension. In this paper, we propose a super WKI hierarchy associated with a $3 \times 3$ matrix spectral problem, in which the first nontrivial member takes the following form:

$$
\begin{aligned}
u_{t}= & i\left[\left(\frac{-u}{\sqrt{1+u v}}\right)_{x}+\frac{2 u \alpha}{1+u v}\left(\frac{\beta}{\sqrt{1+u v}}\right)_{x}\right. \\
& \left.-\frac{u}{\sqrt{1+u v}}\left(\frac{\alpha \beta}{1+u v}\right)_{x}+\frac{2 \alpha \alpha_{x}}{(\sqrt{1+u v})^{3}}\right]_{x}, \\
v_{t}= & i\left[\left(\frac{v}{\sqrt{1+u v}}\right)_{x}+\frac{2 v \beta}{1+u v}\left(\frac{\alpha}{\sqrt{1+u v}}\right)_{x}\right. \\
& \left.+\frac{v}{\sqrt{1+u v}}\left(\frac{\alpha \beta}{1+u v}\right)_{x}-\frac{2 \beta \beta_{x}}{(\sqrt{1+u v})^{3}}\right]_{x},
\end{aligned}
$$




$$
\begin{aligned}
\alpha_{t}= & {\left[\frac{-2}{1+u v}\left(\frac{\alpha}{\sqrt{1+u v}}\right)_{x}-\frac{2 u}{1+u v}\left(\frac{\beta}{\sqrt{1+u v}}\right)_{x}\right.} \\
& +\frac{u \alpha}{1+u v}\left(\frac{v}{\sqrt{1+u v}}\right)_{x}-\frac{\beta}{1+u v}\left(\frac{u}{\sqrt{1+u v}}\right)_{x} \\
& \left.-\frac{3 u \alpha \beta \beta_{x}-(2-u v) \alpha \alpha_{x} \beta}{(\sqrt{1+u v})^{5}}\right]_{x} \\
\beta_{t}= & {\left[\frac{2}{1+u v}\left(\frac{\beta}{\sqrt{1+u v}}\right)_{x}-\frac{2 v}{1+u v}\left(\frac{\alpha}{\sqrt{1+u v}}\right)_{x}\right.} \\
& -\frac{v \beta}{1+u v}\left(\frac{u}{\sqrt{1+u v}}\right)_{x}-\frac{\alpha}{1+u v}\left(\frac{v}{\sqrt{1+u v}}\right)_{x} \\
& +\frac{3 v \alpha \alpha_{x} \beta+(2-u v) \alpha \beta \beta_{x}}{(\sqrt{1+u v})^{5}},
\end{aligned}
$$

which is the well-known WKI equation (1) as $\alpha=0$ and $\beta=0$.

The outline of this paper is as follows. In Section 2, we introduce a $3 \times 3$ matrix spectral problem with two commuting potentials $u$ and $v$, and two anticommuting potentials $\alpha$ and $\beta$. This spectral problem is an extension of the spectral problems associated with the WKI equation. From this spectral problem, a hierarchy of the super WKI equations are proposed with the aid of the zero-curvature equation. In Section 3 , the super bi-Hamiltonian structures of the super WKI hierarchy are constructed by using the super trace identity [22-26]. In Section 4, we derive infinite conservation laws of the super WKI equation by resorting to the spectral parameter expansions. For the applied and analytic aspects on conservation laws, one can refer to [27-30]. We can refer to the two most recent results on the mixed method for the calculation of conservation laws studied in $[29,30]$.

\section{Super WKI Equations}

In this section, a hierarchy of super WKI equations will be obtained. We first introduce a $3 \times 3$ matrix spectral problem:

$$
\begin{aligned}
\phi_{x} & =U \phi, \\
\phi & =\left(\begin{array}{l}
\phi_{1} \\
\phi_{2} \\
\phi_{3}
\end{array}\right), \\
U & =\left(\begin{array}{ccc}
\lambda & \lambda u & \lambda \alpha \\
\lambda v & -\lambda & \lambda \beta \\
-\lambda \beta & \lambda \alpha & 0
\end{array}\right),
\end{aligned}
$$

where $u, v, \lambda, \phi_{1}$, and $\phi_{2}$ are the commuting variables, which can be indicated by the degree $(\bmod 2) p$ as $p(u)=p(v)=$ $p(\lambda)=p\left(\phi_{1}\right)=p\left(\phi_{2}\right)=0 ; \alpha, \beta$, and $\phi_{3}$ are the anticommuting variables which can be indicated by $p$ as $p(\alpha)=p(\beta)=p\left(\phi_{3}\right)$ $=1$. In order to derive the hierarchy of super nonlinear evolution equations associated with the spectral problem (3), we need to solve the stationary zero-curvature equation:

$$
\begin{aligned}
V_{x} & =[U, V], \\
V & =\left(V_{i j}\right)_{3 \times 3},
\end{aligned}
$$

where $p\left(V_{11}\right)=p\left(V_{12}\right)=p\left(V_{21}\right)=p\left(V_{22}\right)=p\left(V_{33}\right)=0 ; \quad p($ $\left.V_{13}\right)=p\left(V_{23}\right)=p\left(V_{31}\right)=p\left(V_{32}\right)=1$. We note that equation (4) is equivalent to

$$
\begin{aligned}
& V_{11 x}=-\lambda v V_{12}+\lambda u V_{21}+\lambda \alpha V_{31}-\lambda \beta V_{13}, \\
& V_{12 x}=2 \lambda V_{12}+\lambda u\left(V_{22}-V_{11}\right)+\lambda \alpha\left(V_{13}+V_{32}\right), \\
& V_{13 x}=\lambda V_{13}+\lambda u V_{23}+\lambda \alpha\left(V_{33}-V_{11}\right)-\lambda \beta V_{12} \\
& V_{21 x}=-2 \lambda V_{21}+\lambda v\left(V_{11}-V_{22}\right)+\lambda \beta\left(V_{31}-V_{23}\right), \\
& V_{22 x}=\lambda v V_{12}-\lambda u V_{21}+\lambda \alpha V_{23}+\lambda \beta V_{32} \\
& V_{23 x}=-\lambda V_{23}+\lambda v V_{13}-\lambda \alpha V_{21}+\lambda \beta\left(V_{33}-V_{22}\right), \\
& V_{31 x}=-\lambda V_{31}-\lambda v V_{32}+\lambda \alpha V_{21}+\lambda \beta\left(V_{33}-V_{11}\right) \\
& V_{32 x}=\lambda V_{32}-\lambda u V_{31}+\lambda \alpha\left(V_{22}-V_{33}\right)-\lambda \beta V_{12}, \\
& V_{33 x}=\lambda \alpha\left(V_{23}+V_{31}\right)+\lambda \beta\left(V_{32}-V_{13}\right)
\end{aligned}
$$

where each entry $V_{i j}=V_{i j}(A, B, C, \rho, \delta)$ is a function of $A, B$, $C, \rho$, and $\delta$ :

$$
\begin{aligned}
& V_{11}=-A, \\
& V_{12}=B, \\
& V_{13}=\delta, \\
& V_{21}=C, \\
& V_{22}=A, \\
& V_{23}=\rho, \\
& V_{31}=-\rho, \\
& V_{32}=\delta, \\
& V_{33}=0,
\end{aligned}
$$

with $p(A)=p(B)=p(C)=0, p(\rho)=p(\delta)=1$. Substituting (6) into (5), we have

$$
\begin{aligned}
& A_{x}=\lambda v B-\lambda u C+\lambda \alpha \rho+\lambda \beta \delta, \\
& B_{x}=2 \lambda B+2 \lambda u A+2 \lambda \alpha \delta, \\
& C_{x}=-2 \lambda C-2 \lambda v A-2 \lambda \beta \rho,
\end{aligned}
$$




$$
\begin{aligned}
& \delta_{x}=\lambda \delta+\lambda u \rho+\lambda \alpha A-\lambda \beta B, \\
& \rho_{x}=-\lambda \rho+\lambda v \delta-\lambda \alpha C-\lambda \beta A .
\end{aligned}
$$

$$
\delta=\sum_{j=0}^{\infty} \delta_{j} \lambda^{-j}
$$

The functions $A, B, C, \rho$, and $\delta$ are expanded as the following Laurent series in $\lambda$ :

$$
\begin{aligned}
& A=\sum_{j=0}^{\infty} A_{j} \lambda^{-j}, \\
& B=\sum_{j=0}^{\infty} B_{j} \lambda^{-j}, \\
& C=\sum_{j=0}^{\infty} C_{j} \lambda^{-j}, \\
& \rho=\sum_{j=0}^{\infty} \rho_{j} \lambda^{-j},
\end{aligned}
$$

Substituting (8) into (7), we can get the Lenard recursion equation as follows:

$$
K G_{j-1}=J G_{j}, J G_{-1}=0, \quad j \geq 0,
$$

where

$$
G_{j-1}=\left(C_{j}, B_{j},-2 \rho_{j}, 2 \delta_{j}, A_{j}\right)^{\mathrm{T}},
$$

and $K$ and $J$ are two operators defined by

$$
\begin{aligned}
K & =\frac{1}{2}\left(K_{i j}\right)_{5 \times 5}, \\
J & =\frac{1}{2}\left(J_{i j}\right)_{5 \times 5},
\end{aligned}
$$

with

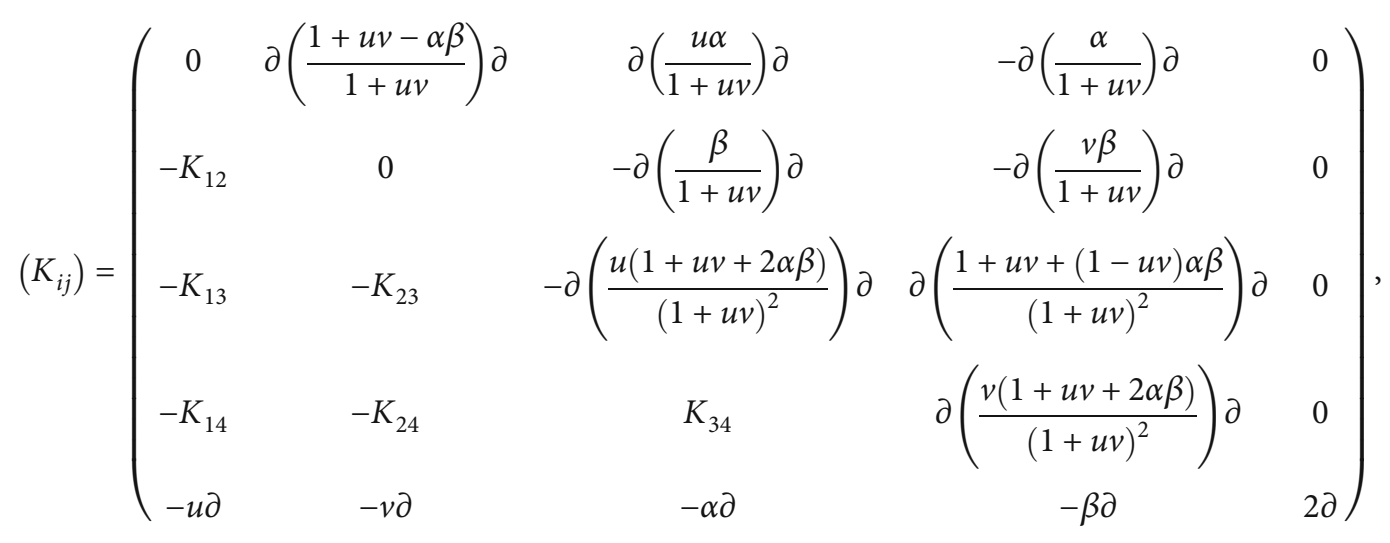

$$
\begin{aligned}
& \left(J_{i j}\right)=\left(\begin{array}{ccccc}
\partial u \partial^{-1} u \partial & 2 \partial+\partial u \partial^{-1} v \partial & \partial u \partial^{-1} \alpha \partial & \partial u \partial^{-1} \beta \partial & 0 \\
2 \partial+\partial v \partial^{-1} u \partial & \partial v \partial^{-1} v \partial & \partial v \partial^{-1} \alpha \partial & \partial v \partial^{-1} \beta \partial & 0 \\
\partial \alpha \partial^{-1} u \partial & \partial \alpha \partial^{-1} v \partial & \partial \alpha \partial^{-1} \alpha \partial & \partial+\partial \alpha \partial^{-1} \beta \partial & 0 \\
\partial \beta \partial^{-1} u \partial & \partial \beta \partial^{-1} v \partial & -\partial+\partial \beta \partial^{-1} \alpha \partial & \partial \beta \partial^{-1} \beta \partial & 0 \\
-u \partial & -v \partial & -\alpha \partial & -\beta \partial & 2 \partial
\end{array}\right) \text {, }
\end{aligned}
$$

and $\partial=\partial_{x}$.

To find a general representation of the solution for (9), we present a Lenard recursion equation as follows:

$$
K g_{j-1}=J g_{j}, \quad j \geq 0,
$$

with condition to identify constants of integration as zero when acting with operator $J^{-1}$ upon $K g_{j}$. This means that $g_{j}$ is uniquely determined by the recursion equation (13). We choose $\operatorname{ker} J=c_{0} g_{-1}=c_{0}(1+u v)^{-3 / 2}(1+u v+\alpha \beta)(-v$, $-u, 2 \beta,-2 \alpha, 1)^{\mathrm{T}}$. Through long calculations, we have 


$$
g_{0}=\left(\begin{array}{c}
\frac{v_{x}}{2(\sqrt{1+u v})^{3}}+\frac{3 v\left(\alpha \beta_{x}-\alpha_{x} \beta\right)-3 v^{2} \alpha \alpha_{x}-(2-u v) \beta \beta_{x}}{2(\sqrt{1+u v})^{5}} \\
\frac{-u_{x}}{2(\sqrt{1+u v})^{3}}+\frac{3 u\left(\alpha \beta_{x}-\alpha_{x} \beta\right)+3 u^{2} \beta \beta_{x}+(2-u v) \alpha \alpha_{x}}{2(\sqrt{1+u v})^{5}} \\
\frac{v_{x} \alpha+2 v \alpha_{x}-2 \beta_{x}}{(\sqrt{1+u v})^{3}}-\frac{3(u v)_{x}(v \alpha-\beta)}{2(\sqrt{1+u v})^{5}} \\
\frac{-u_{x} \beta-2 u \beta_{x}-2 \alpha_{x}}{(\sqrt{1+u v})^{3}}+\frac{3(u v)_{x}(u \beta+\alpha)}{2(\sqrt{1+u v})^{5}} \\
\frac{u v_{x}-u_{x} v}{4(\sqrt{1+u v})^{3}}-\frac{(2-u v)\left(\alpha \beta_{x}-\alpha_{x} \beta\right)-3\left(v \alpha \alpha_{x}-u \beta \beta_{x}\right)}{2(\sqrt{1+u v})^{5}}
\end{array}\right) .
$$

Operating with $\left(J^{-1} K\right)^{j}$ upon $G_{-1}=c_{0} g_{-1}$, we get the general solution of (9).

$$
G_{j}=c_{0} g_{j}+c_{1} g_{j-1}+\cdots+c_{j+1} g_{-1}, \quad j \geq-1,
$$

where $c_{0}, c_{1}, \cdots, c_{j+1}$ are constants of integration and $g_{j}=$ $\left(g_{j}^{(1)}, g_{j}^{(2)}, g_{j}^{(3)}, g_{j}^{(4)}, g_{j}^{(5)}\right)^{\mathrm{T}}$.

Let $\phi$ satisfy the spectral problem (3) and the following auxiliary problem

$$
\phi_{t_{n}}=V^{(n)} \phi
$$

where each entry $V_{i j}^{(n)}=V_{i j}\left(A^{(n)}, B^{(n)}, C^{(n)}, \rho^{(n)}, \delta^{(n)}\right)$ in the matrix $V^{(n)}$ is a polynomial of eigenparameter $\lambda$ with

$$
\begin{aligned}
A^{(n)} & =\sum_{j=0}^{n-1} A_{j} \lambda^{n+1-j}, \\
B^{(n)} & =\sum_{j=0}^{n-1} B_{j} \lambda^{n+1-j}+\lambda\left(B_{n}+u A_{n}\right), \\
C^{(n)} & =\sum_{j=0}^{n-1} C_{j} \lambda^{n+1-j}+\lambda\left(C_{n}+v A_{n}\right), \\
\rho^{(n)} & =\sum_{j=0}^{n-1} \rho_{j} \lambda^{n+1-j}+\lambda\left(\rho_{n}+\beta A_{n}\right), \\
\delta^{(n)} & =\sum_{j=0}^{n-1} \delta_{j} \lambda^{n+1-j}+\lambda\left(\delta_{n}+\alpha A_{n}\right),
\end{aligned}
$$

where $A_{j}, B_{j}, C_{j}, \rho_{j}$, and $\delta_{j}$ are determined by (15). Then, the compatibility condition of (3) and (16) yields the zerocurvature equation $U_{t_{n}}-V_{x}^{(n)}+\left[U, V^{(n)}\right]=0$, which is equivalent to the hierarchy of the super WKI equations.

$$
\left(u_{t_{n}}, v_{t_{n}}, \alpha_{t_{n}}, \beta_{t_{n}}\right)^{\mathrm{T}}=P K G_{n-2}=P J G_{n-1}, \quad n \geq 1,
$$

where $P$ is a projective map $\left(\eta_{1}, \eta_{2}, \eta_{3}, \eta_{4}, \eta_{5}\right)^{\mathrm{T}} \longrightarrow\left(\eta_{1}, \eta_{2}\right.$, $\left.\eta_{3}, \eta_{4}\right)^{\mathrm{T}}$. This can be transformed into

$$
\left(u_{t_{n}}, v_{t_{n}}, \alpha_{t_{n}}, \beta_{t_{n}}\right)^{\mathrm{T}}=c_{0} X_{n-1}+c_{1} X_{n-2}+\cdots+c_{n-1} X_{0}, \quad n \geq 1,
$$

with $X_{j}=P K g_{j-1}=P J g_{j}$. The first nontrivial member in the hierarchy (19) is as follows:

$$
\begin{aligned}
u_{t_{1}}= & \frac{c_{0}}{2}\left[\left(\frac{-u}{\sqrt{1+u v}}\right)_{x}+\frac{2 u \alpha}{1+u v}\left(\frac{\beta}{\sqrt{1+u v}}\right)_{x}\right. \\
& \left.-\frac{u}{\sqrt{1+u v}}\left(\frac{\alpha \beta}{1+u v}\right)_{x}+\frac{2 \alpha \alpha_{x}}{(\sqrt{1+u v})^{3}}\right]_{x} \\
v_{t_{1}}= & \frac{c_{0}}{2}\left[\left(\frac{v}{\sqrt{1+u v}}\right)_{x}+\frac{2 v \beta}{1+u v}\left(\frac{\alpha}{\sqrt{1+u v}}\right)_{x}\right. \\
& \left.+\frac{v}{\sqrt{1+u v}}\left(\frac{\alpha \beta}{1+u v}\right)_{x}-\frac{2 \beta \beta_{x}}{(\sqrt{1+u v})^{3}}\right]_{x} \\
\alpha_{t_{1}}= & \frac{-c_{0}}{2}\left[\frac{2}{1+u v}\left(\frac{\alpha}{\sqrt{1+u v}}\right)_{x}+\frac{2 u}{1+u v}\left(\frac{\beta}{\sqrt{1+u v}}\right)_{x}\right. \\
& -\frac{u \alpha}{1+u v}\left(\frac{v}{\sqrt{1+u v}}\right)_{x}+\frac{\beta}{1+u v}\left(\frac{u}{\sqrt{1+u v}}\right)_{x} \\
\beta_{t_{1}}= & \left.-\frac{3 u \alpha \beta \beta_{x}-(2-u v) \alpha \alpha_{x} \beta}{2}\right]_{x}, \frac{-2}{1+u v}\left(\frac{\beta}{\sqrt{1+u v}}\right)_{x}+\frac{2 v}{1+u v}\left(\frac{\alpha}{\sqrt{1+u v}}\right)_{x} \\
& +\frac{v \beta}{1+u v}\left(\frac{u}{\sqrt{1+u v}}\right)_{x}+\frac{\alpha}{1+u v}\left(\frac{v}{\sqrt{1+u v}}\right)_{x} \\
& \left.\frac{3 v \alpha \alpha_{x} \beta+(2-u v) \alpha \beta \beta_{x}}{(\sqrt{1+u v})^{5}}\right]
\end{aligned}
$$

which is reduced to the famous WKI equation (1) (see [21, 31]) as $t_{1}=t, \alpha=0, \beta=0, c_{0}=2 i$ or the super WKI equation (2) as $t_{1}=t, c_{0}=2 i$. 


\section{Super Bi-Hamiltonian Structures}

In this section, the super bi-Hamiltonian structures of equation (19) will be established by using the super trace identity as follows [23-27]:

$$
\frac{\delta}{\delta U_{0}} \int \operatorname{str}\left(V \frac{\partial U}{\partial \lambda}\right) d x=\lambda^{-\gamma} \frac{\partial}{\partial \lambda}\left(\lambda^{\gamma} \operatorname{str}\left(\frac{\partial U}{\partial U_{0}} V\right)\right)
$$

where $\gamma$ is a constant to be determined and $U_{0}=(u, v, \alpha, \beta)^{T}$. It is easy to observe that

$$
\begin{aligned}
& \operatorname{str}\left(V \frac{\partial U}{\partial \lambda}\right)=-2 A+v B+u C-2 \alpha \rho+2 \beta \delta \\
& \operatorname{str}\left(\frac{\partial U}{\partial u} V\right)=\lambda C \\
& \operatorname{str}\left(\frac{\partial U}{\partial v} V\right)=\lambda B \\
& \operatorname{str}\left(\frac{\partial U}{\partial \alpha} V\right)=-2 \lambda \rho \\
& \operatorname{str}\left(\frac{\partial U}{\partial \beta} V\right)=2 \lambda \delta
\end{aligned}
$$

Substituting (22) and (8) into (21), we arrive at

$$
\begin{aligned}
& \frac{\delta}{\delta U_{0}} \int(-2 A+v B+u C-2 \alpha \rho+2 \beta \delta) d x \\
& =\lambda^{-\gamma} \frac{\partial}{\partial \lambda}\left(\lambda^{\gamma+1}(C, B,-2 \rho, 2 \delta)^{\mathrm{T}}\right), \\
& \frac{\delta}{\delta U_{0}} \int\left(-2 A_{j}+v B_{j}+u C_{j}-2 \alpha \rho_{j}+2 \beta \delta_{j}\right) d x \\
& \quad=(\gamma+1-j)\left(C_{j}, B_{j},-2 \rho_{j}, 2 \delta_{j}\right)^{\mathrm{T}}, \quad j \geq 0 .
\end{aligned}
$$

Through direct calculations, we find that $\gamma=k$ as $c_{k} \neq 0$, $\left\{c_{l}\right\}_{l=0, l \neq k}^{j}=0,0 \leq k \leq j$. Using (23) and noticing (15), we have

$$
\frac{\delta}{\delta U_{0}} H_{j}=\left(C_{j}, B_{j},-2 \rho_{j}, 2 \delta_{j}\right)^{\mathrm{T}}, \quad j \geq 0,
$$

where

$$
H_{0}=-2 c_{0} \int \frac{1+u v-\alpha \beta}{\sqrt{1+u v}} d x
$$

$$
\begin{aligned}
H_{1}= & c_{0} \int\left(\frac{u_{x} v-u v_{x}}{2 u v \sqrt{1+u v}}+\frac{\alpha_{x} \beta-\alpha \beta_{x}+v \alpha \alpha_{x}-u \beta \beta_{x}}{(\sqrt{1+u v})^{3}}\right) d x \\
& -2 c_{1} \int \frac{1+u v-\alpha \beta}{\sqrt{1+u v}} d x, \\
H_{j}= & \sum_{k=0}^{j-2} \frac{c_{k}}{k+1-j} \int\left(\left(u-\partial^{-1} u \partial\right) g_{j-k}^{(1)}+\left(v-\partial^{-1} v \partial\right) g_{j-k}^{(2)}\right. \\
& \left.+\left(\alpha-\partial^{-1} \alpha \partial\right) g_{j-k}^{(3)}+\left(\beta-\partial^{-1} \beta \partial\right) g_{j-k}^{(4)}\right) d x \\
& +c_{j-1} \int\left(\frac{u_{x} v-u v_{x}}{2 u v \sqrt{1+u v}}+\frac{\alpha_{x} \beta-\alpha \beta_{x}+v \alpha \alpha_{x}-u \beta \beta_{x}}{(\sqrt{1+u v})^{3}}\right) d x \\
& -2 c_{j} \int \frac{1+u v-\alpha \beta}{\sqrt{1+u v}} d x, \quad j \geq 2 .
\end{aligned}
$$

From (24), we obtain the desired Hamiltonian form of (19) as follows:

$$
\left(u_{t_{n}}, v_{t_{n}}, \alpha_{t_{n}}, \beta_{t_{n}}\right)^{\mathrm{T}}=K \frac{\delta}{\delta U_{0}} H_{n-1}=J \frac{\delta}{\delta U_{0}} H_{n},
$$

where $K$ and $J$ are two super-Hamiltonian operators defined by

$$
\begin{aligned}
K & =\frac{1}{2}\left(K_{i j}\right)_{4 \times 4}, \\
J & =\frac{1}{2}\left(J_{i j}\right)_{4 \times 4},
\end{aligned}
$$

with $K_{i j}$ and $J_{i j}$ given by (11). Especially, the super WKI equation (20) can be written as follows:

$$
\left(u_{t_{1}}, v_{t_{1}}, \alpha_{t_{1}}, \beta_{t_{1}}\right)^{T}=K \frac{\delta}{\delta U_{0}} H_{0}=J \frac{\delta}{\delta U_{0}} H_{1}
$$

\section{Conservation Laws}

In this section, infinitely, many conservation laws of the super WKI equation (20) will be constructed. First, let us introduce the variables

$$
\begin{gathered}
M=\frac{\phi_{2}}{\phi_{1}}, \\
\Gamma=\frac{\phi_{3}}{\phi_{1}},
\end{gathered}
$$

where $\phi_{1}, \phi_{2}$, and $\phi_{3}$ satisfy (3) and (16) with $n=1$. Noticing that $\phi_{3}^{2}=0$, we get from (3) that

$$
\begin{aligned}
M_{x} & =\lambda\left(v-2 M+\beta \Gamma-u M^{2}-\alpha M \Gamma\right), \\
\Gamma_{x} & =\lambda(-\beta+\alpha M-\Gamma-u M \Gamma) .
\end{aligned}
$$


We expand $M, \Gamma$ in powers of $\lambda^{-1}$ as follows:

$$
\begin{gathered}
M=\sum_{j=0}^{\infty} M_{j} \lambda^{-j}, \\
\Gamma=\sum_{j=0}^{\infty} \Gamma_{j} \lambda^{-j},
\end{gathered}
$$

where $p\left(M_{j}\right)=0, p\left(\Gamma_{j}\right)=1$. Substituting (31) into (30) and comparing the coefficients of the same powers of $\lambda$, we obtain

$$
\begin{aligned}
M_{0} & =\frac{1}{u}\left(-1 \pm \frac{1}{\sqrt{1+u v}}\right), \\
\Gamma_{0} & =\frac{1}{u}\left(\alpha \mp \frac{u \beta+\alpha}{\sqrt{1+u v}}\right),
\end{aligned}
$$

and a recursion formula for $M_{j}$ and $\Gamma_{j}$

$$
\begin{aligned}
M_{j+1}= & \frac{\mp 1}{2 \sqrt{1+u v}}\left(M_{j x}-\Gamma_{0} \Gamma_{j x}+u \sum_{k=1}^{j} M_{k} M_{j-k+1}\right. \\
& \left.-\left(u \Gamma_{0}-\alpha\right) \sum_{k=1}^{j} M_{k} \Gamma_{j-k+1}\right), \\
\Gamma_{j+1}= & \frac{\mp 1}{\sqrt{1+u v}}\left(\Gamma_{j x}-\alpha M_{j+1}+u \sum_{k=0}^{j} M_{j-k+1} \Gamma_{k}\right), \quad j \geq 0 .
\end{aligned}
$$

Since

$$
\frac{\partial}{\partial t} \frac{\phi_{1 x}}{\phi_{1}}=\frac{\partial}{\partial x} \frac{\phi_{1 t}}{\phi_{1}}
$$

$$
\begin{aligned}
I_{0} & = \pm \frac{1+u v-\alpha \beta}{\sqrt{1+u v}} \\
F_{0} & = \pm \frac{c_{0}}{4}\left(\frac{u v_{x}-u_{x} v}{1+u v}-\frac{\left(u v_{x}-u_{x} v\right) \alpha \beta-6 v \alpha \alpha_{x}+6 u \beta \beta_{x}-2(2-u v)\left(\alpha_{x} \beta-\alpha \beta_{x}\right)}{(1+u v)^{2}}\right) .
\end{aligned}
$$

we can derive the conservation law of (20) as follows:

$$
\frac{\partial}{\partial t}(1+u M+\alpha \Gamma) \lambda=\frac{\partial}{\partial x}\left(V_{11}^{(1)}+V_{12}^{(1)} M+V_{13}^{(1)} \Gamma\right)
$$

where

$$
\begin{aligned}
& V_{11}^{(1)}=-c_{0} g_{-1}^{(5)} \lambda^{2}, \\
& V_{12}^{(1)}=c_{0}\left(-u g_{-1}^{(5)} \lambda+g_{0}^{(2)}+u g_{0}^{(5)}\right) \lambda, \\
& V_{13}^{(1)}=c_{0}\left(-\alpha g_{-1}^{(5)} \lambda+\frac{g_{0}^{(4)}}{2}+\alpha g_{0}^{(5)}\right) \lambda .
\end{aligned}
$$

Assuming that $I=1+u M+\alpha \Gamma, \quad F=\left(V_{11}^{(1)}+V_{12}^{(1)} M+\right.$ $\left.V_{13}^{(1)} \Gamma\right) / \lambda$, (35) can be rewritten as $I_{t}=F_{x}$, which is the right form of conservation laws. We expand $I$ and $F$ as series in powers of $\lambda$ with the coefficients which are called conserved densities and currents, respectively

$$
\begin{aligned}
& I=\sum_{j=0}^{\infty} I_{j} \lambda^{-j}, \\
& F=\mp c_{0} \lambda+\sum_{j=0}^{\infty} F_{j} \lambda^{-j},
\end{aligned}
$$

where $c_{0}$ is a integration constant of (15). The first members of conserved densities and currents are as follows:
The recursion relations of $I_{j}$ and $F_{j}(j \geq 1)$ are as follows:

$$
\begin{aligned}
F_{j}= & c_{0}\left(-u g_{-1}^{(5)} M_{j+1}+\left(g_{0}^{(2)}+u g_{0}^{(5)}\right) M_{j}\right. \\
& \left.-\alpha g_{-1}^{(5)} \Gamma_{j+1}+\left(\frac{g_{0}^{(4)}}{2}+\alpha g_{0}^{(5)}\right) \Gamma_{j}\right), \\
I_{j}= & u M_{j}+\alpha \Gamma_{j}, \quad j \geq 1,
\end{aligned}
$$

where $M_{j}$ and $\Gamma_{j}$ can be computed by (33).

\section{Data Availability}

The data used to support the findings of this study are included within the article.

\section{Conflicts of Interest}

The authors declare that they have no conflicts of interest.

\section{Acknowledgments}

This work is supported by the National Natural Science Foundation of China (Grant No. 11871440). 


\section{References}

[1] B. A. Kupershmidt, "A super Korteweg-de Vries equation: an integrable system," Physics Letters A, vol. 102, no. 5-6, pp. 213-215, 1984.

[2] X. Geng and L. Wu, "A new super-extension of the KdV hierarchy," Applied Mathematics Letters, vol. 23, no. 6, pp. 716721, 2010.

[3] P. Mathieu, "Supersymmetric extension of the Korteweg-de Vries equation," Journal of Mathematical Physics, vol. 29, no. 11, pp. 2499-2506, 1988.

[4] M. Gürses and Ö. Oğuz, "A super AKNS scheme," Physics Letters A, vol. 108, no. 9, pp. 437-440, 1985.

[5] M. Gürses and Ö. Oğuz, "A super soliton connection," Letters in Mathematical Physics, vol. 11, no. 3, pp. 235-246, 1986.

[6] Y.S. Li and L. N. Zhang, "Super AKNS scheme and its infinite conserved currents," Il Nuovo Cimento A, vol. 93, no. 2, pp. 175-183, 1986.

[7] Z. Popowicz, "The fully supersymmetric AKNS equations," Journal of Physics A: Mathematical and General, vol. 23, no. 7, pp. 1127-1136, 1990.

[8] Y. I. Manin and A. O. Radul, "A supersymmetric extension of the Kadomtsev-Petviashvili hierarchy," Communications in Mathematical Physics, vol. 98, no. 1, pp. 65-77, 1985.

[9] X. G. Geng and L. H. Wu, "A super extension of Kaup-Newell hierarchy," Communications in Theoretical Physics, vol. 54, no. 4, pp. 594-598, 2010.

[10] X. Geng, B. Xue, and L. Wu, "A super Camassa-Holm equation with N-Peakon solutions," Studies in Applied Mathematics, vol. 130, no. 1, pp. 1-16, 2012.

[11] G. He, Y. Zhai, and X. Geng, "A super hierarchy of the vector nonlinear Schrödinger equations and Hamiltonian structures, conservation laws," Journal of Mathematical Physics, vol. 54, no. 8, article 083509, 2013.

[12] X. Li, "A super extension of Heisenberg hierarchy," International Journal of Theoretical Physics, vol. 53, no. 11, pp. 3765-3773, 2014.

[13] K. Ikeda, "A supersymmetric extension of the Toda lattice hierarchy," Letters in Mathematical Physics, vol. 14, no. 4, pp. 321-328, 1987.

[14] I. Yamanaka and R. Sasaki, "Super Virasoro algebra and solvable supersymmetric quantum field theories," Progress in Theoretical Physics, vol. 79, no. 5, pp. 1167-1184, 1988.

[15] G. H. M. Roelofs and P. H. M. Kersten, "Supersymmetric extensions of the nonlinear Schrödinger equation: symmetries and coverings," Journal of Mathematical Physics, vol. 33, no. 6, pp. 2185-2206, 1992.

[16] C. M. Yung, “The $N=2$ supersymmetric Boussinesq hierarchies," Physics Letters B, vol. 309, no. 1-2, pp. 75-84, 1993.

[17] Q. P. Liu, "Supersymmetric Harry Dym type equations," Journal of Physics A: Mathematical and General, vol. 28, no. 8, pp. L245-L248, 1995.

[18] C. Devchand and J. Schiff, "The supersymmetric CamassaHolm equation and geodesic flow on the superconformal group," Journal of Mathematical Physics, vol. 42, no. 1, pp. 260-273, 2001.

[19] J. C. Brunelli, A. Das, and Z. Popowicz, "Supersymmetric extensions of the Harry Dym hierarchy," Journal of Mathematical Physics, vol. 44, no. 10, pp. 4756-4767, 2003.
[20] S. Q. Lu, X. B. Hu, and Q. P. Liu, "A supersymmetric Ito's equation and its soliton solutions," Journal of the Physical Society of Japan, vol. 75, no. 6, article 064004, 2006.

[21] M. Wadati, K. Konno, and Y. H. Ichikawa, "New integrable nonlinear evolution equations," Journal of the Physical Society of Japan, vol. 47, no. 5, pp. 1698-1700, 1979.

[22] W. X. Ma, J. H. Meng, and H. Q. Zhang, "Integrable couplings, variational identities and Hamiltonian formulations," Global Journal of Mathematical Sciences (GJMS), vol. 1, pp. 1-17, 2012.

[23] W. X. Ma, "Proceedings of the 2nd International Workshop of Nonlinear and Modern Mathematical Physics," AIP Conference Proceedings, vol. 1562, p. 105, 2013.

[24] W. X. Ma, "A soliton hierarchy associated with so(3,R)," Applied Mathematics and Computation, vol. 220, pp. 117122, 2013.

[25] X. B. Hu, "An approach to generate superextensions of integrable systems," Journal of Physics A: Mathematical and General, vol. 30, no. 2, pp. 619-632, 1997.

[26] W. X. Ma, J. S. He, and Z. Y. Qin, "A supertrace identity and its applications to superintegrable systems," Journal of Mathematical Physics, vol. 49, no. 3, article 033511, 2008.

[27] Y. Benia, M. Ruggieri, and A. Scapellato, "Exact solutions for a modified Schrödinger equation," Mathematics, vol. 7, no. 10, p. 908, 2019.

[28] A. Scapellato, "New perspectives in the theory of some function spaces and their applications," AIP Conference Proceedings, vol. 1978, article 140002, 2018.

[29] M. Ruggieri and M. P. Speciale, "Conservation laws by means of a new mixed method," International Journal of Non-Linear Mechanics, vol. 95, pp. 327-332, 2017.

[30] M. Ruggieri and M. P. Speciale, "On the construction of conservation laws: a mixed approach," Journal of Mathematical Physics, vol. 58, no. 2, article 023510, 2017.

[31] G. Z. Tu, "The trace identity, a powerful tool for constructing the Hamiltonian structure of integrable systems," Journal of Mathematical Physics, vol. 30, no. 2, pp. 330-338, 1989. 